\title{
Desorption and Sputtering from Solids Induced by Polyatomic Projectiles
}

\author{
Y. Le Beyec, K. Baudin, A. Brunelle, S. Della-Negra, D. Jacquet, M. Pautrat \\ Institut de Physique Nucléaire, CNRS-IN2P3, F-91406 Orsay Cedex
}

April, 1999

\section{Introduction}

Effects induced by cluster ions colliding with solids have been studied for many years. There is actually a renewal of interest due to new experimental possibilities. Thin film deposition [1] shallow ion implantation [2], surface smoothing and polishing [3] with cluster beams have been developed in several laboratories [4]. Enhanced sputtering with clusters by comparison with single atoms has also been demonstrated long ago by Andersen and Bay [5] with $\mathrm{Te}$ and $\mathrm{Te}_{2}$ projectiles and later on by Beuhler and Friedmann [6] using large water clusters. The secondary emission yield from cluster impact is larger than the sum of the yields of individual atoms. The non linear effects in the emission yield were also measured with $\mathrm{Sb}, \mathrm{Sb}_{2}, \mathrm{Sb}_{3}$ at $30 \mathrm{keV} /$ atom [6] and $\mathrm{Bi}, \mathrm{Bi}_{2}$ at $45 \mathrm{keV} /$ atom [7] bombarding metallic surfaces as Au and Ag. A review on non linear effects in sputtering was recently presented by Andersen [8]. A yield enhancement was also found for secondary ion emission. This was first observed with dimers of $\mathrm{Ne}_{2}$ and $\mathrm{Ar}_{2}$ [9] and with small hydrogen cluster $\mathrm{H}_{n}$ [10]. Large non linear effects in the emission of molecular ions from solid surfaces were an important finding $[11,12]$. These authors emphasized the role of cluster projectiles (as $\mathrm{SF}_{6}$ ) for analytical applications in secondary ion mass spectrometry (SIMS), by comparison with single atomic ions of $\mathrm{Cs}^{+}$or $\mathrm{Xe}^{+}$. In order to investigate the mechanisms of secondary emission of neutrals and ions (clusters and molecular ions) by polyatomic projectiles and clusters, experiments have been performed at Orsay with projectiles over a large energy range (from $\mathrm{keV}$ to $\mathrm{MeV}$ per atom) and various types of targets (metals, insulating material as thin or thick organic layers). Cluster ion sources and accelerators were used. Most of the experiments were made in the event by event mode at the limit of single impacts on the target. Experiments with $\mathrm{keV}$ polyatomic projectiles were also performed at Texas A\&M [13]. Actually cluster beams of $\mathrm{SF}_{5}$ and/or $\left(\mathrm{CO}_{2}\right)$ are being used in SIMS on commercial instruments. The field of mass spectrometry of organic molecules may gain considerably from these developments.

\section{Secondary ion emission with gold cluster projectiles}

Positive gold cluster ions $(\mathrm{Au})_{n}$ with $1 \leq \mathrm{n} \leq 10$ can be easily produced with a liquid metal ion source [14]. A double pulsation technique allows to select a given mass and therefore a single cluster can be chosen as a projectile [14]. The source with its beam line and deflection plates was mounted on a reaction chamber that contains short distance time-of-flight mass spectrometers for secondary ion measurements. The ion emission yield is defined as the ratio between the number of emitted secondary ions and the number of incident projectiles (see ref. [14]). A gold target was bombarded with gold cluster $(\mathrm{Au})_{n}(\mathrm{n}=1-5)$ in the energy range 5 to 60 $\mathrm{keV}$. A non linear effect was observed in the emission of single negative atomic gold ions, induced by impact of $A u_{1}$ to $\mathrm{Au}_{5}$. More spectacular is the emission of gold cluster ions from a solid gold target. It was shown, for example, that the emission of $\left(\mathrm{Au}_{n}\right)^{-}$(with $\left.\mathrm{n} \leq 5\right)$ is several order of magnitude larger with $\mathrm{Au}_{3}$ projectile than with $\mathrm{Au}_{1}$ at $20 \mathrm{keV} /$ atom. These results with a metallic target indicate that the emission of clusters is very much enhanced under impact of cluster projectiles [15]. Insulating materials as CsI targets and organic targets were also bombarded by $\left(A u_{n}\right)$ clusters and polyatomic ions. The secondary ion emission of CsI clusters and of intact molecular ions were severely enhanced with cluster projectiles. The number of constituents in the projectile is a main parameter and the non linear effects are much more important for complex 
secondary ions than for atomic secondary ions. The effect of the number of constituents in projectiles has been easily verified by comparing the emission yield of molecular ions produced by the impact of $\mathrm{C}_{60}$ and $A u_{n}$ at the same velocity. These two projectiles have about the same mass. At the bombarding energy of about 25 $\mathrm{keV}$, the emission yield is more than 5 times larger with $\mathrm{C}_{60}$ than with $\mathrm{Au}_{4}[15]$. Upon impact with the solid the projectile breaks into its constituents and the range of a carbon atom $(\sim 35 \AA)$ is smaller than the range of gold atom $(\sim 160 \AA)$. Therefore the density of energy deposited close to the surface is larger with $\mathrm{C}_{60}$. The secondary emission yield simply reflects the amount of energy that can be released out of the surface. Fig. 1 shows the relative variation of yields of an organic ion $(\mathrm{M}-\mathrm{H})^{-}$from phenylalanine as a function of the energy per unit mass of the projectile $\left(\sim \mathrm{v}^{2}\right)$.

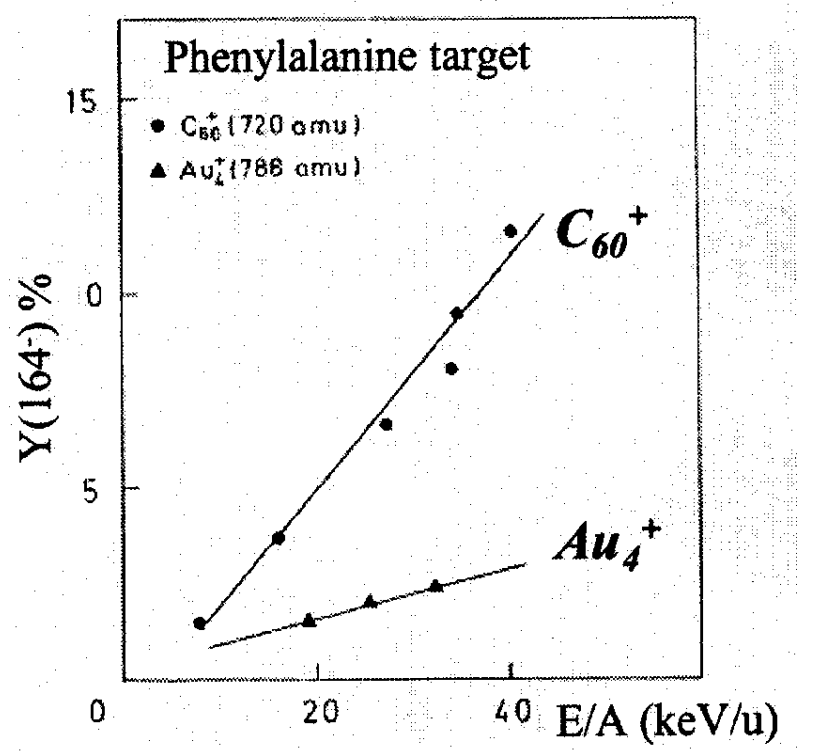

Figure 1. Comparison of emission yields from organic layers after impacts or $\mathrm{C}_{60}$ and $\mathrm{Au}_{4}$ projectiles in the energy range $(5-25 \mathrm{keV})$. The abscissa in $\mathrm{E} / \mathrm{A}(\mathrm{eV} / \mathrm{u})$ is proportional to the square of the projectile incident velocity [15].

\section{Secondary ion emission with organic projectiles}

A fabulous projectile seems to be $\mathrm{C}_{60}$ at several tens of $\mathrm{keV}$, although $\mathrm{C}_{60}$ at several $\mathrm{MeV}$ is now being produced as a beam that induces also very peculiar phenomena in solids [16]. It has been shown that complex polyatomic ions [16] are an excellent probe to analyze thin layers of molecules deposited on various substrates as, for example, contaminant layers of organic compounds. The question of damages created at the sample surface is in some cases important; however, in static
TOF-SIMS, and when using a very low dose (sometimes below $10^{5}$ total) secondary molecular ions can be detected at a sensitivity level not attainable with single atomic projectiles. Organic projectiles $73 \leq \mathrm{m} / \mathrm{z} \leq$ 1200 were used at Orsay in event by event coincidence experiments $[17,18]$ and it was shown that the non linear effect does not extend to large $n$ values ( $n$ being the number of atoms in the projectiles). In fact, above $n$ around 20 the non linear effect vanishes and the secondary emission yield follows a linear dependence with n. A simple apparatus producing molecular ions projectiles with $\mathrm{m} / \mathrm{z}=385$ has been recently tested for secondary ion mass spectrometry of organic compounds [18]. The method is called "spontaneous desorption mass spectrometry" and Fig. 2 shows a comparison of time-of-flight spectra (recorded during the same time) between the measurements in plasma desorption mass spectrometry and in spontaneous desorption mass spectrometry. The sample was a phosphorylated glycolipid $(\mathrm{m} / \mathrm{z}=528.5)$. It is shown here that organic projectiles at a few tens of $\mathrm{keV}$ are efficient for desorption processes.

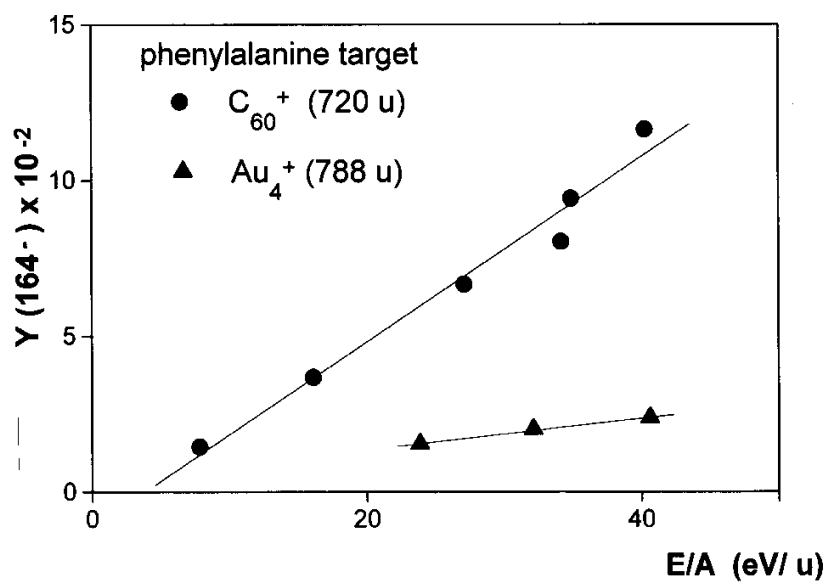

Figure 2. Time-of-flight spectra of $\mathrm{C}_{60}$ obtained by fission fragment impacts from a ${ }^{252} \mathrm{Cf}$ source (a) and the brilliant green spontaneous desorption source (b) [18].

\section{Total sputtering with $\left(\mathrm{A} u_{n}\right)$ projectiles}

As mentioned above the non linear enhancement of secondary ions was also observed for neutral species. Recently, total sputtering yield measurements of gold targets bombarded by $A u_{n}(\mathrm{n}=1$ to 13$)$ clusters were performed in a large energy range from $20 \mathrm{keV}$ to more than $2000 \mathrm{keV}$ per atom. The gold clusters were produced and accelerated by the small tandem accelerator "Aramis" at Orsay and by a 2 MV Van de Graaff at Lyon (Institut de Physique Nucléaire). The mass 
eroded from the target was measured with a quartz micro-balance. A thick quartz crystal was coated with $1000 \mathrm{~nm}$ of gold and a decrease of the gold thickness due to sputtering gives rise to a proportional increase of the oscillation frequency. A sputtering yield as high as 1200 atoms per impact of $A u_{11}$ was measured while a value of only 50 atoms was obtained for single Au projectiles at the same energy per atom $(\sim 100 \mathrm{keV})$. The second main finding of this work was that all the maxima in the sputtering yields do not occur (as expected) at the same velocity of impact of the different $\mathrm{Au}_{n}$ cluster projectiles [19]. Therefore, the strong non-linearity of the emission yield that is observed cannot be given by a simple power law of the nuclear stopping power. With regards to the interpretation of these new results, the shifts of the yield curves maxima towards lower energy per atom for increasing number of constituents in the projectiles is not yet explained. The thermal spike model from Sigmund and Clausen [20] has been applied to try to fit the data. In this model the radius of the spike is a key parameter that should vary both with the number $n$ of atoms and with the energy. This dependence is however not established. These results on total sputtering are now the most complete set of results on the total sputtering of a metal target. A set of results on secondary ion emission has also been obtained with gold clusters in the same energy range as the total sputtering results. The data show that the maxima of yield for molecular ion emission occurs also at relatively low energy with respect to the maxima of the nuclear stopping power [21]. For organic material the maxima of yield with $\mathrm{Au}_{4}$ is around $80 \mathrm{keV} /$ atom. This is well illustrated in Fig. 3 that shows the yield variation of $(\mathrm{M}-\mathrm{H})^{-}$from a phenylalanine sample as a function of the energy per atom of $\mathrm{Au}_{n}$ projectiles. The same shape of yield curves with a peak named as "the sugar loaf peak" is observed with different types of samples (organic and inorganic). These new results demonstrate that the projectile energy range of clusters between 40 and $120 \mathrm{keV} /$ atom are of great importance since "resonance" effects may occur in the solid, that lead to an important release of matter from the surface. There are also possible applications with enhanced efficiency and sensitivity for surface and bulk analysis.

\section{Energetic MeV clusters}

It must be recalled also that increasing the energy of cluster projectiles might be useful for applications. Both aspects of pulverisation and production of thin layers by redeposition as well as material modification of materials may gained from the increase of energy. Surface analysis and depth profiling are only some aspects of secondary ion mass spectrometry. For example
$\mathrm{C}_{60}$ projectiles at $20 \mathrm{MeV}$ are very efficient to emit and identify intact molecular ions of mass larger than 25000 u or large clusters. A time-of-flight mass spectrum can be recorded with a good statistics with only $10^{4}$ incident ions since the emission yield of very large molecules is much more than one secondary ion/impact [22]. For example the desorption yield of insulin molecules by 20 $\mathrm{MeV} \mathrm{C}_{60}$ as shown in Fig. 4 is about 100 times larger than with $\mathrm{MeV}$ atomic ion projectiles. The desorption of molecules with masses above $100.000 \mathrm{u}$ could also be investigated in the future. Some surfaces are strongly modified in one impact, others are not. Fast clusters can create in metals [23] permanent defects which are not produced by single ions having the same energy loss. Whatever the energy loss domain (nuclear or electronic) it is the amount of energy density deposited in the material which is important for the induced effects.

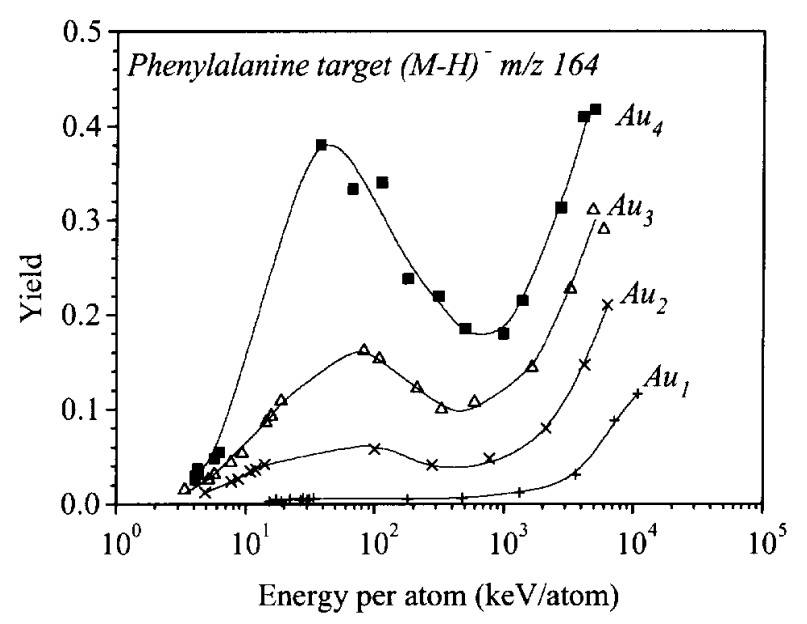

Figure 3. Desorption yield of the ion $(\mathrm{M}-\mathrm{H})^{-}$from phenylalanine layers $(\sim 2000 \AA)$ bombarded by gold clusters $A u_{n}$ projectiles over a large energy range per atom. It is clearly observed the importance of the so-called "sugar loaf peak" at the energy per atom around $60 \mathrm{keV}$.

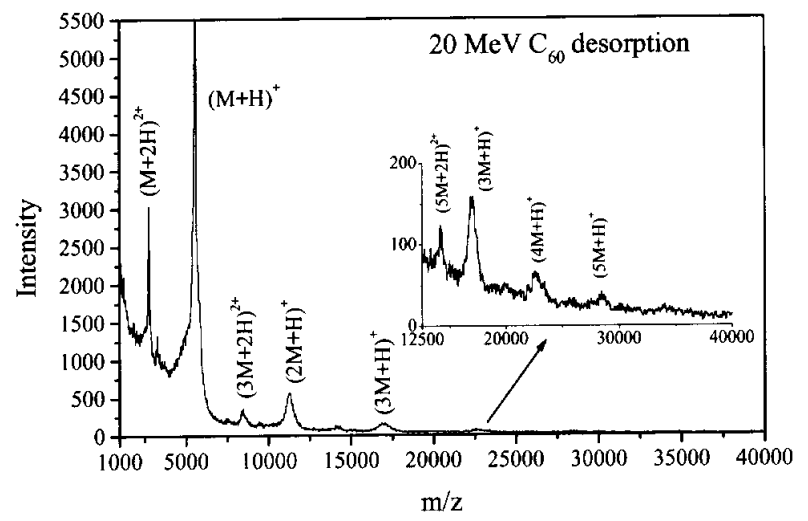

Figure 4. Example of PDMS-type time-of-flight spectra obtained by the impact of $20 \mathrm{MeV} \mathrm{C}_{60}$ projectiles on an insulin sample. A spectrum with good statistics can be obtained in a few minutes of beam. 


\section{Conclusion}

When using polyatomic ions as projectiles the enhancement of emission yield of secondary ions and neutrals is clearly established in a large range of incident energies. The larger the secondary ions the more important the non linear effects. Damage cross sections must be considered but at projectile energies below $50 \mathrm{keV}$ and for organic samples the rate of emission seems to be larger than the rate of damages. Molecules and clusters which are not seen with single atomic projectiles can be easily observed with polyatomic projectiles. The use of fast complex projectiles has considerably broadened the field of collisions of ions with solids in fundamental research. With atomic projectiles it has been shown for many years that the velocity was an important parameter. Fast atomic ions, in the electronic regime of energy loss, were the most efficient for desorbing intact molecular ions from solid samples. It appears now that polyatomic projectiles at much lower velocities seem to be even more efficient. $\mathrm{MeV}$ ion beams require sophisticated equipment and the use of fission fragments from ${ }^{252} \mathrm{Cf}$ is rather limited (and sometimes restricted). From the results presented here, cluster ions at a few tens of $\mathrm{keV} /$ atom are certainly promising. In the future the use of large multiply charged clusters or molecules may also be a unique way to study phenomena related to a high energy deposition in solids.

\section{References}

[1] I. Yamada et al, Z. Phys. D3, 137 (1986).

[2] I. Yamada et al, Similarities and differences between atomic nuclei and clusters, AIP conference 416, 310 (1997), edited by Y. Abe, I. Arai, S.M. Lee, K. Yabana

[3] P. Henkes, R. Kiligelhfer, J. Phys. (Paris) 50, C2, 159 (1989), J. Gspann, Similarities and differences between atomic nuclei and clusters, AIP Conference 416, 299 (1997).

[4] See also proceedings of conferences - $\mathrm{MeV}$, keV ions and cluster interactions with surfaces and materials, J. Phys. C2 (1989), Edited by Y.Le Beyec, S. DellaNegra, J.P.Thomas. - Polyatomic ion impact on solids and related phenomena, Nucl. Instr. and Meth. B88, 12 (1994), proceedings edited by Y. Le Beyec, Y. Hoppilliard, H. Bernas. - Correlated effects in atomic and cluster ion bombardment and implantation, EMRS Con- ference, Proceedings in Nucl. Instr. and Meth. B112 (1996). - Nanometric phenomena induced by laser, ion and cluster beams, EMRS Conf., NIM B123 (1997). Review : Y. Le Beyec, Int. J. of Mass Spectrom. and Ion Processes 174 (1998) 101-117

[5] H.H. Andersen and H.L. Bay, J. Appl. Phys. 45, 953 (1974).

[6] R.J. Beuhler and L. Friedman, J. Appl. Phys. 48, 3928 (1977).

[7] D.A. Thompson and S.S. Johar, Appl. Phys. Letters 34, 342 (1979).

[8] H.H. Andersen, in "Sputt 92", The Royal Danish Academy of Sciences and Letters, Matematisk-Fysiske Meddelelser 43, 127 (1993).

[9] K. Wittmack, Surf. Science 90, 557 (1979).

[10] J.P. Thomas et al, Phys. Rev. Lett. 55, 103 (1985).

[11] A.D. Appehans and J.Delmore, Analytical Chem. 61, 1087 (1989).

[12] W. Szymczak, K. Wittmaack, Nucl. Instr. and Meth. in Phys. Res. B88, 149 (1994). W. Szymezak and K. Wittmaack, in: Methods and Mechanisms for Producing Ions from Large Molecules, eds. K.G. Standing and W. Ens, NATO ASI Ser. B: Physics vol. 269 (Plenum, New York and London, 1991) p. 123.

[13] M.G. Blain et al, J. Phys. C2, 845 (1989). M.J. Van Stipdonk et al, Rapid. Com. in Mass Spectrom. 10, 1987 (1996).

[14] M. Benguerba et al, Nucl. Instr. and Meth. B62, 8 (1991).

[15] K. Baudin et al, NIM B88 (1994) 160 and K. Baudin, Thesis University Paris XI (1993).

[16] S. Della-Negra et al, Nucl. Instr. and Meth. B74, 453 (1993).

[17] M.G. Blain et al, J. Phys. C2, 147 (1989).

[18] K. Baudin et al, Rapid. Comm. in Mass Spectrom. 12, 111 (1998).

[19] H.H. Andersen et al, Phys. Rev. Letters 80, 5433 (1998). A. Brunelle, H.H. Andersen et al, to be published

[20] P. Sigmund and C. Clausen, J. Appl. Phys. A33, 141 (1984).

[21] K. Baudin, A. Brunelle, S. Della-Negra, J. Depauw, D. Jacquet, Y. Le Beyec, to be published

[22] A. Brunelle et al, Int. J. of Mass Spectrom. and Ion Processes 164, 143 (1997).

[23] H. Dammak et al, Phys. Rev. Lett. 74, 1135 (1995). 